\title{
Prevalencia moderada de pie en riesgo de ulceración en diabéticos tipo 2 según IGWDF en el contexto de la atención primaria
}

Dante Rodríguez Alonso 1, a, Fredix Mercedes Chávez 1, b, David Rodríguez Díaz 2, c, Tania Polo López 2, c, Ángela Rivera Begazo 2, c, Eddy Margarita Guzmán Yparraguirre 2,c

\section{RESUMEN}

Objetivo: Determinar la prevalencia del pie en riesgo de ulceración (PRU) según International Working Group on Diabetic Foot (IWGDF) expresada por la presencia de neuropatía diabética periférica (NPD) y/o enfermedad arterial periférica (EAP) y/o deformidad (D) y sus factores asociados en diabéticos tipo 2 en la atención primaria de la salud (APS) de Trujillo.

Materiales y métodos: Se captó una muestra de 301 diabéticos tipo 2 de centros de salud, públicos y privados del distrito de Trujillo, pertenecientes a la APS; posteriormente se estratificó según el sistema IWGDF para pie en riesgo, se consideraron diagnósticos de NPD, EAP y D. Se realizó estadística descriptiva y analítica con las variables evaluadas por SPSS versión 22. Además, se solicitó una acreditación de un comité de ética e investigación.

Resultados: Los diabéticos tipo 2 mostraron los siguientes diagnósticos: el 13,3 \% presentaron NPD, EAP en 18,6 \% y D en $64,1 \%$. Además, se encontró que el $86,7 \%$ no presentan PRU, el $4 \%$ se encuentra en un riesgo leve y el $9,3 \%$ en moderado; con una prevalencia total de 13,3\%. Finalmente, se encontraron como factores de riesgo asociados a la regularidad de atención, hipertensión arterial, hipoglicemia, actividad física y calzado ortopédico.

Conclusiones: Estudio epidemiológico único en la APS peruana que muestra una prevalencia de nivel moderado en PRU, pero menor respecto a sus similares latinoamericanos, siendo el componente deformidad la más frecuentes; y con factores asociados positivos y muy débiles cuando se analizó los pacientes con y sin riesgo.

Palabras clave: Prevalencia; Pie; Diabetes mellitus tipo 2; Factores de riesgo (Fuente: DeCS BIREME).

\section{Moderate prevalence of foot ulceration risk according to the IWGDF guidelines in type 2 diabetic patients attending the primary health care}

\section{ABSTRACT}

Objective: To determine the prevalence of foot ulceration risk (FUR) according to the International Working Group on the Diabetic Foot (IWGDF) guidelines, demonstrated by the presence of peripheral diabetic neuropathy (PDN) and/or peripheral arterial disease (PAD) and/or deformity (D), as well as its associated factors, in type 2 diabetic patients attending the primary health care (PHC) in Trujillo.

Materials and methods: A sample of 301 type 2 diabetic patients was collected from public and private health centers of the district of Trujillo belonging to the PHC. Subsequently, said sample was stratified according to the IWGDF guidelines on foot at risk, considering diagnoses such as PDN, PAD and D. Descriptive and analytical statistics were performed with the variables evaluated by the IBM SPSS Statistics V22.0. In addition, accreditation was requested from an ethics and research committee.

Results: Type 2 diabetic patients showed the following diagnoses: $13.3 \%$ presented PDN, $18.6 \%$ PAD and $64.1 \%$ D. Besides, it was found that $86.7 \%$ have no FUR, $4 \%$ are at slight risk of and $9.3 \%$ are at moderate risk of developing foot ulcers, with a total prevalence of $13.3 \%$. Finally, the associated risk factors were medical attention frequency, hypertension, hypoglycemia, physical activity and orthopedic footwear.

Conclusions: This is the only epidemiological study performed in the Peruvian PHC showing a moderate prevalence of FUR, but lower than its Latin-American peers' experiences. Deformity was the most frequent component, and positive and very weak associated factors were found when analyzing patients with and without risk factors.

Keywords: Prevalence; Foot; Diabetes mellitus type 2, Risk factors (Source: MeSH NLM).

1. Centro Médico Privado Ortocentro, Unidad de investigación. Trujillo, Perú

2. Universidad César Vallejo, Vicerrectorado de investigación. Trujillo, Perú.

a. Master en Investigación clínica, Médico especialista en pie diabético.

b. Bachiller en Medicina y Especialista en Medicina Familiar y Comunitaria.

c. Docente investigador. 


\section{INTRODUCCIÓN}

La diabetes mellitus está considerada dentro de las principales causas de morbimortalidad mundial dentro de las enfermedades crónicas en adultos según International Diabetes Federation y una de sus principales complicaciones la patología el pie diabético, la insuficiencia renal crónica y la retinopatía diabética $(1,2)$. Según reportes de Ministerio de Salud peruano, el pie diabético y la neuropatía periférica diabética son las principales causas de complicación en diabetes, con $30 \%$ y $7 \%$ de prevalencia respectivamente, y se encuentran en la costa norte del Perú ${ }^{(3)}$.

El síndrome pie diabético es una infección, ulceración o destrucción de partes blandas del pie y tobillo asociada con neuropatía y/o enfermedad arterial periférica en un paciente diabético, y las lesiones frecuentemente resultan de una combinación de muchos factores de riesgo que puede llevar a úlcera, amputación o muerte (4).

Debido a este enorme problema para categorizar los riesgos que llevan al pie diabético, se utilizan sistemas de diagnósticos tales como la Scottish Intercollegiate Guideline Network (SIGN), la ADA (American Diabetes Association), la NHS (National Health Service) Borders Foot Classification System y la International Working Group on the Diabetic Foot (IWGDF); esta última, la más aceptada por consenso mundial ${ }^{(5,6)}$.

La estratificación de estos sistemas sobre diagnóstico ha llevado al uso del término pie en riesgo de ulceración (PRU), el cual denomina a aquellos pacientes diabéticos que presentan neuropatía diabética periférica $\mathrm{y} / \mathrm{o}$ enfermedad arterial periférica y/o deformidad de pie sin haber presentado aún úlcera en el pie e incorporado por IWGDF como estándar para uso internacional; además, su identificación lleva a un diagnóstico precoz que permiten intervenciones médicas tempranas preventivas farmacológicas simples ${ }^{(7)}$.

Dentro de los factores de riesgo asociados a PRU se encuentran los demográficos, antecedentes de la diabetes mellitus, estilo de vida, comorbilidades, antecedentes quirúrgicos de pie, cuidados de pies; y los factores desencadenantes son la neuropatía diabética periférica, la enfermedad arterial periférica y la deformidad musculoesquelética del pie ${ }^{(8)}$.

La neuropatía periférica diabética (NPD) es la presencia de síntomas y/o signos de disfunción del nervio periférico de los miembros inferiores en diabéticos después de la exclusión de otras causas ${ }^{(9)}$. La enfermedad arterial periférica diabética (EAP) es el síndrome caracterizado por alteración macrovascular de las arterias sin afectación coronaria, aórtica ni cerebral en diabéticos (10). La deformidad músculoesquelética del pie es el síndrome caracterizado por deformidad flexible o rígida que abarca desde el antepié al retropié de origen idiopático sin causa congénita o secundaria (11).

El sistema diagnóstico de la IWGDF considera como prueba neurológica (PN) los síntomas como adormecimiento u otros, la evaluación táctil con el monofilamento de $10 \mathrm{~g}$, y la evaluación de la vibración con el diapasón $128 \mathrm{~Hz}$; la prueba arterial (PA) considera los síntomas de claudicación, la palpación de pulsos de la arteria tibial posterior y arteria pedia, uso de detector de doppler en las arterias mencionadas y el cálculo del índice brazo tobillo de cualquiera de las arterias mencionadas; y la prueba musculoesquelética (PMSK) considera la deformidades fijas del ante pie y la deformidad en el arco plantar interno por podoscopia ${ }^{(12)}$.

La clasificación IWGDF describe como grado 0 sin NPD o EAP o DMSK o úlcera, grado 1 con NPD sin EAP ni DMSK, grado 2 con NPD, EAP y DMSK; y grado 3 con antecedente de úlcera o úlcera en pie; se considera los grados 0 y 1 como de bajo riesgo y los grado 2 y 3 como de alto riesgo ${ }^{(13)}$.

Las experiencias más significativas en Latinoamérica en PRU diabética en la atención primaria de la salud fueron la de Bortoletto et al. en Brasil con una prevalencia de 27,9 \% evaluadas por el sistema IWGDF, Álvarez y col. en Cuba 56 \% evaluadas por el sistema IWGDF, y Cuevas et al. en Ecuador con prevalencia de 69 \% con el sistema SIGN ${ }^{(14-16)}$. A nivel de la atención secundaria de la salud, se encontró el estudio de Damas-Casani et al. en Perú evaluados con el sistema IWGDF con una prevalencia de $70 \%{ }^{(17)}$.

Debido a la alta prevalencia de diabetes en la costa norte del Perú, la frecuencia del pie diabético como una importante complicación de la enfermedad, la ausencia de estudios epidemiológicos acerca del pie en riesgo a úlcera a nivel nacional y la intención de identificar precozmente este cuadro para prevenirlo; se justificó determinar la prevalencia del pie en riesgo a úlcera y los factores asociados en pacientes diabéticos realizado en el nivel primario de atención de salud con el sistema diagnóstico de la IGWDF en pacientes diabéticos tipo 2 captados de centros de salud públicos y evaluados en el centro especializado Ortocentro de la provincia de Trujillo. 


\section{MATERIALES Y MÉTODOS}

Se realizó una evaluación de 301 pacientes diabéticos atendidos en el programa de estrategia sanitaria de diabetes de una unidad de pie diabético, captados por el sistema ambulatorio privado (Centro médico Ortocentro categorizado como 1-2 por SuSalud) de la ciudad de Trujillo entre los meses de marzo y diciembre del 2017; y procedentes de centros de salud púbicos y privados compuestos de la siguiente manera: 100 pacientes para MINSA, 151 pacientes para EsSalud, y 50 pacientes para privados .

Los pacientes cumplían los criterios de inclusión establecidos para la presente investigación: ser diabéticos tipo 2 según los indicadores nacionales del programa de diabetes del Ministerio de Salud peruano, no tener úlceras en el pie o antecedentes de esta condición, no sufrir enfermedad mental o discapacidad motora que impida movilizarse, u otras patologías neurológicas secundarias. Todos los pacientes fueron evaluados por la técnica de entrevista -observación siguiendo una ficha de recolección denominada "Pie en riesgo a ulceración en la atención primaria de Salud según el sistema IWGDF"; que considera la anamnesis, examen clínico de los aspectos neurológico periférico, arterial periférico y musculoesquelético, los criterios diagnósticos y la categorización del PRU según la IWGDF (Tabla 1).

Tabla 1. Categorización de pie en riesgo a ulceración según IWGDF

\begin{tabular}{cll} 
Categoría & \multicolumn{1}{c}{ Componentes } & \multicolumn{1}{c}{ Riesgo } \\
\hline 0 & Sin NPD & Ninguno \\
1 & Con NPD & Leve \\
2 & Con NPD y EAP y/o D & Moderado \\
3 & Antecedente de úlcera o amputación & Alto \\
\hline
\end{tabular}

NPD: Neuropatía periférica diabética, EAP: Enfermedad arterial periférica, D: Deformidad.

La aplicación de la ficha de recolección de datos fue realizada por el personal de enfermería y médico, entrenados durante un periodo de 3 meses por el equipo de investigadores y expertos. Este proceso se dio en dos fases:

Primera parte: Anamnesis, donde se consideró aspectos demográficos, antecedentes clínicos de la diabetes (tiempo de enfermedad, tipo de tratamiento, regularidad de control, profesional que atiende), complicaciones de diabetes, estilo de vida, cuidados del pie, síntoma de adormecimiento, síntoma de claudicación y síntomas de deformidad o callos. Para el caso de regularidad del control médico se consideró controlado si el paciente acudía a la consulta y se controlaba la glucosa por lo menos cada 3 meses a un programa de salud público o privado de diabetes.

Segunda parte: Examen físico general y específico que incluye la evaluación neurológica según la sensibilidad táctil (St) con monofilamento de $10 \mathrm{~g}$ y sensibilidad vibratoria (Sv) con diapasón de $128 \mathrm{~Hz}$; evaluación arterial periférica según el cálculo del índice tobillo - brazo (ITB), previa identificación de la arteria pedia, y la evaluación de la deformidad por la inspección de callos o deformidades en antepié y medio-retropié en el podoscopio.

Luego se analizaron los datos para diagnóstico de la neuropatía periférica diabética (NPD), enfermedad arterial periférica (EAP), deformidad musculoesquelético (D) y categorización de pie en riesgo fueron realizados por el investigador principal siguiendo los parámetros del consenso de la IWGDF para la neuropatía periférica diabética (sensibilidad táctil o sensibilidad propioceptiva alterada), para enfermedad arterial periférica (índice de tobillo brazo menor de 0,9 y mayor de 1,3 ) y para la deformidad del pie (alteración mínima de dos parámetros como la presencia de callos o deformidades del ante pie o deformidad del medio pie).

En los componentes éticos y deontología, se solicitó el consentimiento informado por escrito a todos los pacientes; se les explicó el beneficio de ser diagnosticado por pruebas no invasivas, simples y 
exactas. Adicionalmente, se presentó el proyecto a un comité de ética e investigación nacional acreditada, siendo aprobado por el Comité de Ética e Investigación del Hospital San Bartolomé de Lima con la resolución del 12 de febrero del 2017.

Además, se trabajó con estadísticas descriptivas para identificar la frecuencia relativa, frecuencias absolutas, promedio y desviación estándar para las variables cuantitativas y cualitativos respectivamente. Finalmente, se utilizó estadística analítica como la prueba de Chi cuadrado, para buscar asociación de cada variable entre pacientes con y sin riesgo a pie ulcerado según el software estadístico SPSS versión 22 .

\section{RESULTADOS}

Los pacientes diabéticos tipo 2 evaluados mostraron las siguientes características epidemiológicas: del total de evaluados el 57,1 \% correspondió al grupo etario 50 - 69 años y predominó el género femenino con $59,5 \%$. En cuanto a las características clínicas de la diabetes se encuentran los factores asociados: con tratamiento antidiabéticos orales con un predominante del $93,7 \%$, el control médico regular predominó con $81,7 \%, 5$ a más años con presencia de la enfermedad en $61,5 \%$; y el $42,5 \%$ tienen presencia de HTA. Otro aspecto valorado fue los estilos de vida, se halló un alto porcentaje asociado a la actividad física $(74,1 \%)$, un $50,2 \%$ se relaciona con la dieta para diabéticos, el 27,9 \% con la retinopatía, el $20,6 \%$ con hipoglicemia, el $83,4 \%$ con los cuidados podológicos, el $70,4 \%$ se vincula con la ausencia de calzado ortopédico y el 39,9\% con el índice de masa corporal, correspondiente al sobrepeso (Tabla 2).

Tabla 2. Características generales de pacientes diabéticos evaluados para diagnóstico de pie en riesgo para ulceración en la atención primaria de la salud -Trujillo

\begin{tabular}{|c|c|c|c|}
\hline Características & Factores epidemiológicos & f & $\%$ \\
\hline \multirow[t]{7}{*}{ Demográficas } & Grupo etario & & \\
\hline & 30-49 años & 33 & 11 \\
\hline & 50-69 años & 172 & 57,1 \\
\hline & 70 a más años & 96 & 31,9 \\
\hline & Género & & \\
\hline & Femenino & 179 & 59,5 \\
\hline & Masculino & 122 & 40,5 \\
\hline \multirow{15}{*}{$\begin{array}{l}\text { Antecedentes clínicos } \\
\text { de la diabetes }\end{array}$} & Tiempo de enfermedad & & \\
\hline & Menor de 5 años & 116 & 38,5 \\
\hline & 5 a más años & 185 & 61,5 \\
\hline & Tipo de tratamiento & & \\
\hline & Antidiabéticos orales & 282 & 93,7 \\
\hline & Insulina & 19 & 6,3 \\
\hline & Profesional que atiende & & \\
\hline & General & 186 & 61,8 \\
\hline & Especialista & 115 & 38,2 \\
\hline & Control médico & & \\
\hline & Regular & 246 & 81,7 \\
\hline & Irregular & 55 & 18,3 \\
\hline & Hipertensión arterial & & \\
\hline & Sí & 128 & 42,5 \\
\hline & No & 173 & 57,5 \\
\hline
\end{tabular}


Dante Rodríguez Alonso, Fredix Mercedes Chávez, David Rodríguez Díaz, Tania Polo López, Ángela Rivera Begazo, Eddy Margarita Guzmán Yparraguirre

\begin{tabular}{|c|c|c|c|}
\hline Características & Factores epidemiológicos & f & $\%$ \\
\hline \multirow{18}{*}{$\begin{array}{l}\text { Complicaciones de } \\
\text { diabetes }\end{array}$} & \multicolumn{3}{|l|}{ Nefropatía } \\
\hline & Sí & 33 & 11 \\
\hline & No & 268 & 89 \\
\hline & \multicolumn{3}{|l|}{ Retinopatía } \\
\hline & Sí & 84 & 27,9 \\
\hline & No & 217 & 73,1 \\
\hline & \multicolumn{3}{|l|}{ Hipoglicemia } \\
\hline & Sí & 62 & 20,6 \\
\hline & No & 239 & 79,4 \\
\hline & \multicolumn{3}{|l|}{ Coma hiperosmolar } \\
\hline & Sí & 12 & 4 \\
\hline & No & 289 & 96 \\
\hline & \multicolumn{3}{|l|}{ Infarto de miocardio } \\
\hline & Sí & 16 & 5,3 \\
\hline & No & 285 & 94,7 \\
\hline & \multicolumn{3}{|l|}{ Enfermedad cerebrovascular } \\
\hline & Sí & 31 & 10,3 \\
\hline & No & 270 & 89,7 \\
\hline \multirow[t]{15}{*}{ Estilos de vida } & \multicolumn{3}{|l|}{ Actividad física } \\
\hline & Sí & 223 & 74,1 \\
\hline & No & 78 & 25,9 \\
\hline & \multicolumn{3}{|l|}{ Dieta para diabético } \\
\hline & Sí & 151 & 50,2 \\
\hline & No & 150 & 49,8 \\
\hline & \multicolumn{3}{|l|}{ Cuidados podológicos } \\
\hline & Sí & 251 & 83,4 \\
\hline & No & 50 & 16,6 \\
\hline & \multicolumn{3}{|l|}{ Calzado ortopédico } \\
\hline & Sí & 89 & 29,6 \\
\hline & No & 212 & 70,4 \\
\hline & \multicolumn{3}{|l|}{ Consumo de alcohol } \\
\hline & Sí & 29 & 9,6 \\
\hline & No & 272 & 90,4 \\
\hline \multirow[t]{4}{*}{ Examen general } & \multicolumn{3}{|l|}{ Índice de masa corporal } \\
\hline & Normal & 104 & 34,6 \\
\hline & Sobrepeso & 120 & 39,9 \\
\hline & Obeso & 77 & 25,6 \\
\hline
\end{tabular}

*Según International Working Group on diabetic foot.

En lo que respecta al examen clínico, se encontró un predominio de $52,2 \%$ en aquellos pacientes que presentan el síntoma de adormecimiento, el 10,3\% sensibilidad táctil, 6,6\% con sensibilidad vibratoria, el $18,6 \%$ con índice tobillo-brazo (ITB) y la deformidad en $64,1 \%$. Por otro lado, la vulnerabilidad vinculada con el diagnóstico de NPD fue de $13,3 \%$ de diabéticos, el 18,6 $\%$ con EAP y, según el diagnóstico de deformidad, fue $64,1 \%$. Con respecto a la clasificación de pie diabético en riesgo según sistema de IWGDF se encontró que el $86,7 \%$ no presentar ningún riesgo, el $4 \%$ presentan un riesgo leve y el 9,3\% moderado (Tabla 3). 
Prevalencia moderada de pie en riesgo de ulceración en diabéticos tipo 2 según IGWDF en el contexto de la atención primaria

Tabla 3. Características clínicas, diagnóstico y estratificación de pacientes diabéticos evaluados para diagnóstico de pie en riesgo para ulceración en la atención primaria de la salud -Trujillo

\begin{tabular}{|c|c|c|c|}
\hline Criterios & Parámetro & f & $\%$ \\
\hline \multirow[t]{9}{*}{ Síntomas } & \multicolumn{3}{|l|}{ Adormecimiento } \\
\hline & Sí & 157 & 52,2 \\
\hline & No & 144 & 47,8 \\
\hline & \multicolumn{3}{|l|}{ Claudicación } \\
\hline & Sí & 138 & 45,8 \\
\hline & No & 163 & 54,2 \\
\hline & \multicolumn{3}{|l|}{ Presencia de callos o deformidad } \\
\hline & Sí & 148 & 49,2 \\
\hline & No & 153 & 50,8 \\
\hline \multirow[t]{12}{*}{ Hallazgos clínicos } & \multicolumn{3}{|l|}{ Sensibilidad táctil (St) } \\
\hline & Alterado & 31 & 10,3 \\
\hline & Conservado & 270 & 89,7 \\
\hline & \multicolumn{3}{|l|}{ Sensibilidad vibratoria (Sv) } \\
\hline & Alterado & 20 & 6,6 \\
\hline & Conservado & 281 & 93,4 \\
\hline & \multicolumn{3}{|l|}{ Índice tobillo-brazo (ITB) } \\
\hline & Alterado & 56 & 18,6 \\
\hline & Normal & 245 & 81,4 \\
\hline & \multicolumn{3}{|l|}{ Deformidad (D) } \\
\hline & Sí & 193 & 64,1 \\
\hline & No & 108 & 35,9 \\
\hline \multirow[t]{9}{*}{ Diagnósticos } & \multicolumn{3}{|l|}{ Neuropatía periférica (NP: St y/o Sp) } \\
\hline & Sí & 40 & 13,3 \\
\hline & No & 261 & 86,7 \\
\hline & \multicolumn{3}{|l|}{ Enfermedad arterial periférica (EAP: ITB) } \\
\hline & Sí & 56 & 18,6 \\
\hline & No & 245 & 81,4 \\
\hline & \multicolumn{3}{|l|}{ Deformidad(D) } \\
\hline & Sí & 193 & 64,1 \\
\hline & No & 108 & 35,9 \\
\hline \multirow[t]{3}{*}{ Pie en riesgo } & Ninguno Leve (NP) & 261 & 86,7 \\
\hline & \multirow[t]{2}{*}{ Moderado (NP y EAP y/o D) } & 12 & 4 \\
\hline & & 28 & 9,3 \\
\hline
\end{tabular}

* Según la International Working Group on Diabetic Foot

Finalmente, se dividió a la muestra total en dos grupos, diabéticos con y sin riesgo, con 40 y 261 casos con el propósito de realizar el análisis de los factores epidemiológicos más importantes; para lo cual se utilizó la prueba Chi cuadrado; se encontró una asociación significativa con factores como regularidad de atención médica, presencia de hipertensión arterial, presencia de hipoglicemia, actividad física presente y calzado ortopédico ausente con valores $p$ de $0,04,0,02,0,04$, $0,03,0,02$ respectivamente; además, con un valor alfa de 0,05 y coeficiente de contingencia con correlación positivas de muy baja intensidad, pues lo-s valores oscilan entre 0,01 - 0,19 (Tabla 4). 
Dante Rodríguez Alonso, Fredix Mercedes Chávez, David Rodríguez Díaz, Tania Polo López, Ángela Rivera Begazo, Eddy Margarita Guzmán Yparraguirre

Tabla 4. Factores epidemiológicos asociados evaluados con y sin pie en riesgo para ulceración en la atención primaria de la salud Trujillo

\begin{tabular}{|c|c|c|c|c|}
\hline $\begin{array}{c}\text { Factor } \\
\text { epidemiológico }\end{array}$ & Parámetro & $\begin{array}{l}\text { Sin PR } \\
(n: 261)\end{array}$ & $\begin{array}{c}\text { Con PR } \\
(\mathrm{n}: 40)\end{array}$ & Valor $p$ \\
\hline & & f & f & $\alpha: 0,05$ \\
\hline \multirow[t]{7}{*}{ Demográficas } & Grupo etario & & & 0,72 \\
\hline & 30-49 años & 30 & 3 & \\
\hline & 50-69 años & 149 & 23 & \\
\hline & 70 a más años & 82 & 14 & \\
\hline & Género & & & 0,94 \\
\hline & Femenino & 155 & 24 & \\
\hline & Masculino & 106 & 16 & \\
\hline \multirow{12}{*}{$\begin{array}{l}\text { Clínicos de la } \\
\text { diabetes }\end{array}$} & Tiempo de enfermedad & & & 0,12 \\
\hline & Menor de 5 años & 105 & 11 & \\
\hline & 5 a más años & 156 & 29 & \\
\hline & Tipo de tratamiento & & & 0,74 \\
\hline & Antidiabéticos orales & 245 & 37 & \\
\hline & Insulina & 16 & 3 & \\
\hline & Control médico ${ }^{1}$ & & & 0,04 \\
\hline & Presente & 218 & 28 & \\
\hline & Ausente & 43 & 12 & \\
\hline & Hipertensión arterial ${ }^{2}$ & & & 0,02 \\
\hline & Sí & 118 & 10 & \\
\hline & No & 143 & 30 & \\
\hline \multirow{9}{*}{$\begin{array}{l}\text { Complicaciones de } \\
\text { diabetes }\end{array}$} & Retinopatía & & & 0,28 \\
\hline & Sí & 70 & 14 & \\
\hline & No & 191 & 26 & \\
\hline & Hipoglicemia $^{3}$ & & & 0,04 \\
\hline & Sí & 49 & 13 & \\
\hline & No & 212 & 27 & \\
\hline & Nefropatía & & & 0,38 \\
\hline & Sí & 27 & 6 & \\
\hline & No & 234 & 34 & \\
\hline \multirow[t]{12}{*}{ Estilos de vida } & Actividad física ${ }^{4}$ & & & 0,03 \\
\hline & Sí & 199 & 24 & \\
\hline & No & 62 & 16 & \\
\hline & Dieta para diabético & & & 0,29 \\
\hline & Sí & 134 & 17 & \\
\hline & No & 127 & 23 & \\
\hline & Cuidados podológicos & & & 0,87 \\
\hline & Sí & 218 & 33 & \\
\hline & No & 43 & 7 & \\
\hline & Calzado ortopédico 5 & & & 0,02 \\
\hline & $S i^{*}$ & 71 & 18 & \\
\hline & No & 190 & 22 & \\
\hline
\end{tabular}


Prevalencia moderada de pie en riesgo de ulceración en diabéticos tipo 2 según IGWDF en el contexto de la atención primaria

\begin{tabular}{|c|c|c|c|c|}
\hline $\begin{array}{c}\text { Factor } \\
\text { epidemiológico }\end{array}$ & Parámetro & $\begin{array}{l}\text { Sin PR } \\
(n: 261)\end{array}$ & $\begin{array}{c}\text { Con PR } \\
(n: 40)\end{array}$ & Valor $p$ \\
\hline & & f & f & $\alpha: 0,05$ \\
\hline & Consumo de alcohol & & & 0,29 \\
\hline & Sí & 27 & 2 & \\
\hline & No & 234 & 38 & \\
\hline \multirow[t]{4}{*}{ Examen general } & Índice de masa corporal & & & 0,88 \\
\hline & Normal & 90 & 14 & \\
\hline & Sobrepeso & 103 & 17 & \\
\hline & Obeso & 68 & 9 & \\
\hline \multirow[t]{9}{*}{ Síntoma } & Adormecimiento & & & 0,52 \\
\hline & Sí & 138 & 19 & \\
\hline & No & 123 & 21 & \\
\hline & Claudicación & & & 0,82 \\
\hline & Sí & 119 & 19 & \\
\hline & No & 142 & 21 & \\
\hline & Presencia de callos o deformidad & & & 0,06 \\
\hline & Sí & 128 & 20 & \\
\hline & No & 133 & 20 & \\
\hline
\end{tabular}

NP: neuropatía periférica, $C$ (coeficiente de contingencia): ${ }^{1}: 0,12,{ }^{2}: 0,14,{ }^{3}: 0,11,{ }^{4}: 0,13,50,13$

*Según la International Working Group on Diabetic Foot

\section{DISCUSIÓN}

El sistema de estratificación del PRU, implementada por la IWGDF, tiene como clave la neuropatía periférica; debido a que es la primera patología presente en la fisiopatología del pie diabético ${ }^{(18)}$. Es por ello que la implementación de esta estrategia de evaluación, del pie en riesgo, está dirigida fundamentalmente a la atención primaria de la salud; ya que mientras más precoz sea menor probabilidad de presentarse la úlcera, según Abramczyk ${ }^{(19)}$.

Según la Organización Mundial de la Salud (20); el sistema de salud de Latinoamérica tiene una atención primaria de la salud muy similar, caracterizada por la poca implementación y, en el caso de la diabetes no es diferente; es por ello que dirigimos nuestra investigación a su prevalencia y factores de riesgo en este contexto.

En torno a esto encontramos que la prevalencia de pie en riesgo es del 13,3\%, sin considerar el riesgo alto. Este valor se encuentra por debajo de los hallados en otros estudios latinoamericanos como es el caso de Bortolleto et al. y Santos et al. en el país de Brasil que identificaron una prevalencia $25,2 \%$ y $16 \%$ respectivamente; además, en Cuba, Álvarez et al. encontraron valores de $42,9 \%$ y en Ecuador, Brutto et al., un $27 \%{ }^{(14,15,21,22)}$. Cabe resaltar que los porcentajes encontrado por Santos et. al se aproximan a nuestro estudio, esto debería a la metodología que utilizó; la cual fue muy similar en tamaño de muestra, sistema de clasificación, validador y centro de evaluación.

Cuando analizamos basados en los criterios de pie en riesgo como NPD, EAP, y deformidad del pie encontramos que:

- Para el caso de la NPD, nuestro resultado de 13,3\% estuvo por debajo de los estudios que precisaron este dato, tales como Álvarez et al. y Brutto et al. con valores de $63,7 \%$ y $59 \%$ respectivamente. Esto se explica a las acciones de educación y monitoreo de nuestra población de diabéticos por el servicio público o privado, manifestado por la alta frecuencia de regularidad en la atención y control metabólico.

- En cuanto a EAP, nuestro resultado de $18,6 \%$ se encontró por debajo de los estudios realizados por Álvarez et al. y Brutto et al., quienes identificaron prevalencias de $29,7 \%$ y $50 \%$ respectivamente; esto se debería a que nuestra población presentaba 
una alta frecuencia de actividad física, mediana frecuencia de hipertensión arterial y pocas comorbilidades.

- Por lo que se refiere a la deformidad del pie, hallamos una prevalencia del $63 \%$; la cual estuvo por encima de otros estudios como los realizados por Álvarez et al. y Brutto et. al.; quienes encontraron valores de $42,9 \%$ y $23,6 \%$ respectivamente. Esto se explica porque el alto nivel de exploración del evaluador y validador, quienes precisaron más signos de deformidad del sistema musculoesquelético, y dieron prioridad a la presencia de rigidez, callos, deformidades y alteración del arco plantar.

- Otro aspecto explorado en este estudio fue el análisis de diferentes factores asociados con pie en riesgo y sin riesgo como el aspecto demográfico, antecedentes de diabetes, enfermedades concomitantes, estilos de vida, cuidados de pies y otros; donde encontraron valores significativos y correlaciones positivas de muy baja intensidad $(0.01$ - 0.19).

- Los valores de prevalencia encontrados relacionados con la regularidad de control médico e hipertensión arterial, fue similar a los estudios de Santos et al. y Bortoletto et al.; esto fue debido a que el mejor control metabólico evita el progreso de la enfermedad, tal como se afirma en una revisión sistemática realizada por Monteiro -Soaires et. al ${ }^{(6)}$.

- Además de lo antes mencionado, la presencia de actividad física es un factor para la presencia o no de la enfermedad; lo encontrado coincide con el estudio de Bortolletto et al. Esto se debería a que el movimiento, en el diabético, provoca mejora en la fisiología de los nervios y emociones con lo que se evita la presentación de complicaciones, sobre todo la NPD, como afirma Crews et al. en una revisión crítica ${ }^{(23)}$.

- Algo a resaltar en el presente estudio es la identificación como factores asociados la presencia de hipoglicemia y la ausencia del calzado ortopédico; que no se habrían analizado o considerado en ningún estudio regional mencionado previamente. La hipoglicemia, probablemente se explica por la poca experiencia del personal médico de atención primaria de salud con el uso de antidiabéticos orales, como muestra un estudio de Sämann et al (24). Así mismo existen investigaciones, como la de Pereira-Despaigne et al., que demostrarían que el calzado inadecuado está relacionado con pie en riesgo ${ }^{(25)}$.

Es importante mencionar que las limitaciones encontradas para el estudio son las dificultades para contar con la HBa1c en más de la mitad de los casos, pues es poco frecuente el uso de este parámetro en la atención primaria de la salud; además, existió un sesgo de selección de la población que, por estrategia, formaban parte de un programa de EsSalud y la práctica privada; sin embargo, se contó con personal calificado de alta calidad que evaluó y validó los procedimientos.

En conclusión, nuestra investigación científica es un estudio epidemiológico único en la atención primaria de la salud peruana; se encontró una prevalencia moderada del PRU, según el sistema IWGDF, y menor en prevalencia respecto a sus similares latinoamericanos. La deformidad fue el componente del PRU más frecuentes de los tres. Además, se hallaron factores asociados positivos y muy débiles como la regularidad de control médico, hipertensión arterial, hipoglicemia, actividad física y uso de calzado ortopédico. El sistema de diagnóstico de la IWGDF para PRU debe ser aplicado en el contexto de la atención primaria del Perú como prioridad para evitar las amputaciones.

\section{REFERENCIAS BIBLIOGRÁFICAS}

1. Cavan D, Fernandes J, Makaroff L, Ogurtsova K, Webber S. Diabetes Atlas. 7th. ed. New York: International diabetes Federation; 2015:24-31. https://www.idf.org/e-library/ epidemiology-research/diabetes-atlas.html

2. Grupo para el consenso de pie diabético. Guía de pie diabético. Revista del ALAD. 2010; 18(2):73-84. http://www. alad-americalatina.org/documentos-de-consenso-de-laalad/.

3. Ramos W, López T, Revilla L, More L, Huamani M, Pozo M. Resultados de la vigilancia epidemiológica de Diabetes mellitus en hospitales notificantes del Perú, 2012. Rev Peru Med Exp Salud Pública. 2014; 31(1):9-15.

4. Rodríguez D. Manual médico quirúrgico de pie diabético experiencia de la costa norte del Perú. 1ra. ed. Trujillo: Colegio Médico del Perú; 2014:3-6.

5. Monteiro-Soares M, Vaz-Carneiro A, Sampaio S, DinisRibeiro M. Validation and comparison of currently available stratification systems for patients with diabetes by risk of foot ulcer Development. Eur J Endocrinol. 2012; 167(3): 401 407.

6. Monteiro-Soares M, Boyko EJ, Ribeiro J, Ribeiro I, DinisRibeiro M. Risk stratification systems for diabetic foot ulcers: a systematic review. Diabetologia. 2011; 54:1190-1199.

7. Monteiro-Soares M, Boyko EJ, Ribeiro J, Ribeiro I, DinisRibeiro M. Predictive factors for diabetic foot ulceration: a systematic review. Diabetes Metab Res Rev. 2012; 28(7):574600.

8. Alonso-Fernández M, Mediavilla-Bravo JJ, López-Simarro F, 
Comas-Samper JM, Carramiñana-Barrera F, Mancero-Romero $\mathrm{J}$, et al . Evaluación de la realización del cribado del pie diabético en Atención Primaria. Endocrinol Nutr. 2014; 61(6): 311-317.

9. Boulton A, Vileikyte A. Painful Diabetic Neuropathy in Clinical Practice. 1st. ed. Heidenberg: Springer; 2011:1-39.

10. Schaper NC, Andros G, Apelqvist J, Bakker K, Lammer J, Lepantalo $M$, et al. Specific guidelines for the diagnosis and treatment of peripheral arterial disease in a patient with diabetes and ulceration of the foot 2011. Diabetes Metab Res Rev. 2012; 28(1): 236-237.

11. Formosa C, Gatt A and Chockalingam N. The importance of clinical biomechanical assessment of foot deformity and joint mobility in people living with type- 2 diabetes within a primary care setting. Prim Care Diabetes. 2013; 7(1): 45-50.

12. Hinchliffe RJ, Brownrigg JR, Apelqvist J, Boyko EJ, Fitridge $\mathrm{R}$, Mills JL, et al. IWGDF guidance on the diagnosis, prognosis and management of peripheral artery disease in patients with foot ulcers in diabetes. Diabetes Metab Res Rev. 2016 ;32(1):37-44.

13. Schaper NC, Van Netten JJ, Apelqvist J, Lipsky BA, Bakker K; International Working Group on the Diabetic Foot. Prevention and management of foot problems in diabetes: a Summary Guidance for Daily Practice 2015, based on the IWGDF Guidance Documents. Diabetes Metab Res Rev. 2016; 32 (1):715.

14. Bortoletto MS, De Andrade SM, Matsuo T, Haddad Mdo C, González AD, Silva AM. Risk factors for foot ulcers-A cross sectional survey from a primary care setting in Brazil. Prim Care Diabetes. 2014; 8(1): 71-76.

15. Álvarez Seijas E, Mena Bouza K ,Faget Cepero O, Coneza Gonzales Al, Dominguez Alonso E. El pie de riesgo de acuerdo con su estratificación en pacientes con diabetes mellitus. Rev Cubana Endocrinol. 2015; 26(2):158-171.

16. Cueva Recalde JF. Validación del puntaje de riesgo para ulcera en pie diabético del SIGN en un grupo de pacientes en Quito. Av Diabetol. 2009; 25:486-90.

17. Damas-Casani VA, Yovera-Aldana $M$, Seclén Santisteban S. Clasificación de pie en riesgo de ulceración según el Sistema IWGDF y factores asociados en pacientes con diabetes mellitus tipo 2 de un hospital peruano. Rev Med Hered. 2017; 28(1):512.

18. Bus SA, van Netten JJ, Lavery LA, Monteiro Soares M, Rasmussen A, Jubiz Y, et al. IWGDF guidance on the prevention of foot ulcers in at-risk patients with diabetes. Diabetes Metab Res Rev. 2016;32 (1):16-24.

19. Abramczyk A. Results of specialized ambulatory diabetes care among diabetes patients at the level of primary health care--in the light of nationwide research. Adv Clin Exp Med. 2012;21(1):63-8

20. Arredondo A, Azar A, Recamán AL. Diabetes, a global public health challenge with a high epidemiological and economic burden on health systems in Latin America. Glob Public Health. 2018;13(7):780-787.

21. Del Brutto OH, Mera RM, King NR, Zambrano M, Sullivan LJ. The burden of diabetes-related foot disorders in communitydwellers living in rural Ecuador: Results of the Atahualpa Project. Foot. 2016; 28:26-29.

22. Santos Thomazelli F, Machado C, Dolçan K. Análise do risco de pé diabético em umambulatório interdisciplinar de diabetes. Rev AMRIGS. 2015; 59(1):10-14.

23. Crews RT, Schneider KL, Yalla SV, Reeves ND, Vileikyte L. Physiological and psychological challenges of increasing physical activity and exercise in patients at risk of diabetic foot ulcers: a critical review. Diabetes Metab Res Rev. 2016;32(8):791-804.

24. Sämann A, Lehmann T, Heller T, Müller N, Hartmann P, Wolf $\mathrm{GB}$, Müller UA. A retrospective study on the incidence and risk factors of severe hypoglycemia in primary care. Fam Pract. 2013;30(3):290-3.

25. Pereira Despaigne OL, Palay Despaigne MS, Frómeta Ríos V, Neyra Barrios RM. Efectividad de un programa educativo en pacientes con pie diabético de riesgo. MEDISAN. 2015;19(1):69-77.

Fuentes de financiamiento:

Este artículo ha sido financiado por los autores.

Conflictos de interés:

Los autores declaran no tener ningún conflicto de interés.

\section{Correspondencia:}

Dante Rodríguez Alonso

Dirección: Av. América Sur 384, Urb. Palermo. Trujillo, Perú.

Teléfono: 05144242164

Correo electrónico: ortocentro30@gmail.com

Recibido: 02 de abril de 2018

Evaluado: 13 de abril de 2018

Aprobado: 14 de mayo de 2018

(c) La revista. Publicado por Universidad de San Martín de Porres, Perú. (cc) Br Licencia de Creative Commons Artículo en acceso abierto bajo términos de Licencia Creative Commons Atribución 4.0 Internacional. (http://creativecommons.org/licenses/by/4.0/)

ORCID iDs

Dante Rodríguez Alonso Fredix Mercedes Chávez David Rodríguez Díaz Tania Polo López Ángela Rivera Begazo (iD) https://orcid.org/0000-0002-6662-9210 (iD) https://orcid.org/0000-0001-8820-6991 (iD) https://orcid.org/0000-0002-9203-3576 (iD) https://orcid.org/0000-0003-3285-4222 (D) https: //orcid.org/0000-0002-9211-224X Eddy Margarita Guzmán Yparraguirre (iD https://orcid.org/0000-0001-8438-6219 